\title{
Modelling of Permeation Grouting considering Grout Self-Gravity Effect: Theoretical and Experimental Study
}

\author{
Yanbin Fu $\mathbb{D}^{\mathbb{D}},{ }^{1,2,3}$ Xiuling Wang $\mathbb{D}^{2},{ }^{2}$ Sizhan Zhang $\mathbb{D}^{1},{ }^{1}$ and Yong Yang $\mathbb{D}^{4}$ \\ ${ }^{1}$ College of Civil and Transportation Engineering, Shenzhen University, Shenzhen, Guangdong 518060, China \\ ${ }^{2}$ Key Laboratory for Special Area Highway Engineering of Ministry of Education, Chang'an University, Xi'an, \\ Shaanxi 710064, China \\ ${ }^{3}$ Underground Polis Academy of Shenzhen University, Shenzhen, Guangdong 518060, China \\ ${ }^{4}$ JiZhunFangZhong Chengdu Architectural Design Co., Ltd., Chengdu, Sichuan 610000, China
}

Correspondence should be addressed to Xiuling Wang; wangxiuling@chd.edu.cn

Received 5 August 2019; Revised 28 October 2019; Accepted 5 November 2019; Published 27 November 2019

Academic Editor: Alain Portavoce

Copyright (c) 2019 Yanbin Fu et al. This is an open access article distributed under the Creative Commons Attribution License, which permits unrestricted use, distribution, and reproduction in any medium, provided the original work is properly cited.

Self-gravity is one key parameter for behavior characterization of grout permeation and diffusion. This study proposes mathematical models for permeation grouting with consideration of grout self-gravity effect. The models concerning power law, Bingham, and Newtonian grouts are based on the generalized Darcy's law and spherical diffusion theory. In addition, a prediction model of grout concretion dimension used for Bingham grout was developed. An analysis of the injection pressure distribution law and a comparative evaluation of diffusion radius considering self-gravity effect using established models were conducted subsequently. Moreover, grouting experiments were performed to check and verify the prediction model. The experimental results showed that injection pressure decreases linearly with increase of diffusion radius for the power-law grout, while nonlinear decrease of injection pressure was confirmed in Bingham and Newtonian grouts in this case. Three grouts approximately diffuse in an "ellipsoidal" shape, and it is confirmed that the diffusion radius is closely related to grout self-gravity. The Newtonian grout produces the maximum diffusion radius compared with the other two grouts whether the gravity effects were considered or not. The grout quantity under a smaller water-to-cement $(w / c)$ ratio exhibits a significant difference and undergoes two increasing stages, whereas the quantity simply tends to be stable after it reaches its maximum in terms of the larger $(w / c)$ ratios. The constructed dimension prediction model agrees well with the experimental results, which can be helpful for design and assessment of the grouting scheme.

\section{Introduction}

Grouting technique has been extensively used in soil reinforcement, water plugging, and deviation rectification of buildings as well as other engineering practices [1-6]. Based on grout diffusion behaviors and mechanism, three methods are commonly used for grouting activities, compaction grouting [7], fracture grouting [8-11], and permeation grouting [12]. Though multiple forms of grout diffusion occur simultaneously in injected soil, one of them often plays a leading role under certain conditions. For example, materials with large porosity in the sandy layer produce the larger internal pore passages that engender less inhibitory effect during grout flow, which is favorable for effective implementation of the permeation grouting technique $[13,14]$. In terms of the utilization of the grout materials, the cement slurry now is most commonly used for grouting practices as a result of low cost compared with other grout materials. In reality, the variations of injected soils and grout materials as well as the uncertainty of external environment always yield challenges for estimation works on grout permeation and diffusion.

To promote grouting technique in civil engineering, many researchers have released the studies on grouting materials $[15,16]$, grouting performance [17-19], and grouting device [20]. Mollamahmutoglu and Yilmaz [21] firstly evaluated some basic properties of the superfine cement suspensions such as stability, viscosity, and setting 
time, and then permeability behaviors under different grouting pressures of the studied suspensions injected into sand specimens with different gradations were examined. Markou and Droudakis [22] evaluated some relevant parameters influencing grout effectiveness by an experimental investigation. The results confirmed that several parameters such as $(w / c)$ ratio, bleed capacity of suspensions, and permeability coefficient of used sands actually control the grouted sand properties so as to determine grout quality. Huang et al. [23] developed a novel method of vacuum grouting to overcome shortcomings such as insufficient diffusion radius due to low grouting pressure. Then, a vacuum modification model is proposed after conducting a modification for boundary conditions of Maag's spherical diffusion model. Kim et al. [24] put forward a method and corresponding equipment with oscillatory injection so as to improve depth of the grout permeation. In this study, the clogging theory was utilized to evaluate enhancement effect of the groutability, and the results indicated that usage of the developed equipment increased grout volume and permeation depth by more than $30 \%$ and $25 \%$, respectively.

A series of studies are concentrated on modelling for grouting behavior, and three grout constitutive equations (i.e., Newtonian fluid, Bingham fluid, and power-law fluid) were most commonly used for grouting theory analysis $[25,26]$. Yoon and El Mohtar [27] proposed a filtration model used to estimate maximum permeation distance in bentonite grouting, and the equation based on yield stress can effectively obtain the maximum distance at a high yield stress. Maghous et al. [28] established a macroscopic model where cement grout flows in the porous medium. The model is presented on the basis of the mass balance equations and filtration law, which can be utilized to investigate the behaviors of the radial flow filtration. In view of the impacts of variations of grout viscosity on grouting evaluation, Zhang et al. [29] developed a step-wise calculation method considering uneven distribution of grout viscosity, in which circular grouting area was divided into minimal annular units. They further studied the distribution law of grout viscosity and pressure in the grouting zone. Some necessary simplifications and assumptions have to be made for compromising with difficulties on accurate definition of complicated process of slurry diffusion $[30,31]$, resulting in considerable deviation in modelling of actual practices. Though a great deal of efforts from scholars and researchers have been made in model modification [32-34], few investigations have been made to model the behaviors of the permeation grouting by taking into account the self-gravity effect of the grouts. The grout diffusion in rock or soil is generally affected by injection pressure, physical properties of grout (e.g., grout self-gravity), and injected medium. The grout gravity is an important factor for mechanical analysis on grouting engineering, as it plays an increasingly dominant role with the attenuation of injection pressure. Taking into account the self-gravity effect in modelling can provide a more accurate prediction on slurry diffusion. The effect is more significant when grout diffuses at a vertical direction. This consideration may also eliminate uncertainty in practices $[35,36]$. For example, the slurry gravity in backfill grouting of shield segments leads to a larger pressure at the top grouting hole than that at the bottom hole $[37,38]$. Therefore, it is urgent to focus on this issue, which could provide more useful information to relevant research and engineering practice.

In this paper, the generalized Darcy's law has been utilized to derive grout diffusion models considering impacts of the self-gravity on permeation behaviors for power law, Bingham, and Newtonian grouts and followed by a dimension prediction model of grout concretion. To validate the proposed dimension prediction model, novel experimental equipment including the model box system, the grouting system, the pressure system, and the measurement system has been developed. The proposed model is well checked by the grouting experiments that allow grout flowing through a sand medium as the modeled and measured results are in good agreement with each other.

\section{Mathematical Modelling for Slurry Permeation and Diffusion}

2.1. General and Basic Assumptions. The grout diffusion in the sandy layer is considered as permeation predominates. As shown in Figure 1, the point-source-based grout generally diffuses in an approximate spherical shape [39]. The grout flows through the grouting pipe to injected soil, and then diffuses around from bottom of the grouting pipe through pore passages of the soils, along with attenuation of grouting pressure. The grout actually diffuses in a curving path as a result of inhomogeneity of soil pores. However, assuming grout diffuses in a straight path is always required for convenient modelling and analysis.

The flowing velocity is regarded as a key parameter to judge grout states; however, it is difficult to be determined. Some previous findings suggested that grout permeates in the laminar flow state when it is injected into the ground. Regarding the spherical diffusion model, the pores of soil layers can be considered as radial capillary tubes from center of sphere outward [35, 40,41]. The size of capillary channels in the sandy layer varies significantly, and it is extremely difficult to reveal the real state through a mathematical model. For convenience, we assume the capillary channels into the tubes with the same radius in modelling. The Dupuit-Forchheimer relation was introduced to transform the permeation model in single tubes into the macroscopic state, and then the generalized Darcy's law (applicable to laminar flow) is applied to derive the mathematical model on the basis of the capillary group theory [42]. Therefore, consider a laminar incompressible grout of finite length $\mathrm{d} l$ and radius $r$ in a circular tube of radius $r_{0}$ (Figure 2). Further, we assume that microelement shares the same axis with the tube, and $\alpha$ is the angle between the grout flow direction and the $x$-axis, which varies from 0 to $2 \pi$ anticlockwise.

Before formulation of the model, the following basic assumptions are introduced: (1) the sand and soil that grout being injected into are isotropic and homogeneous; (2) the grout is in power law, Bingham, and Newtonian fluids, respectively; (3) the grout is grouted into soil at the bottom of grouting pipes; and (4) the grout diffuses in a spherical shape. 


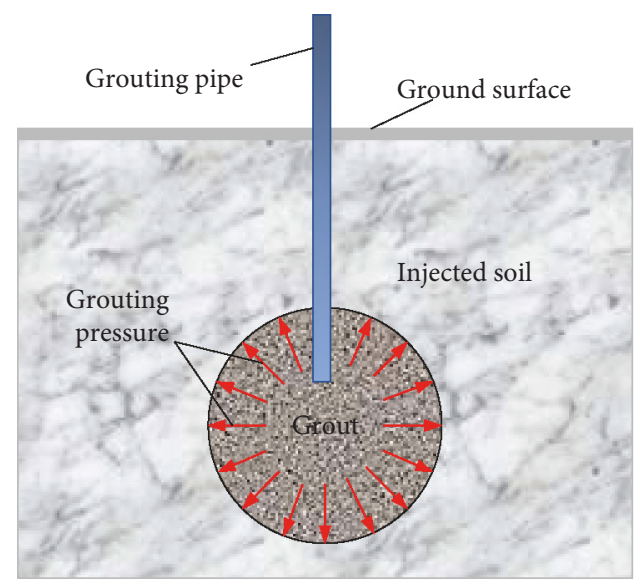

(a)

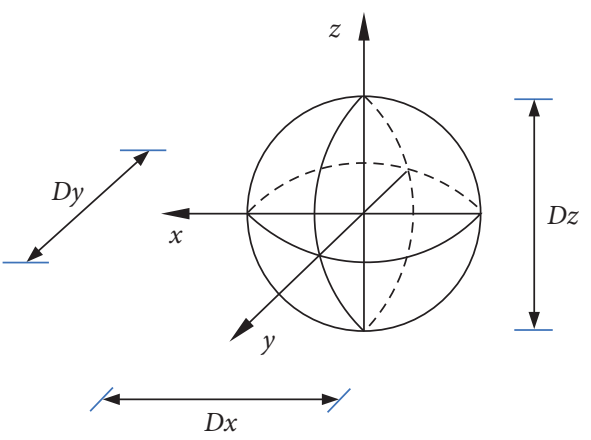

(b)

FIGURE 1: Diagram of the (a) grout permeation diffusion and (b) direction indication of grout concretion.

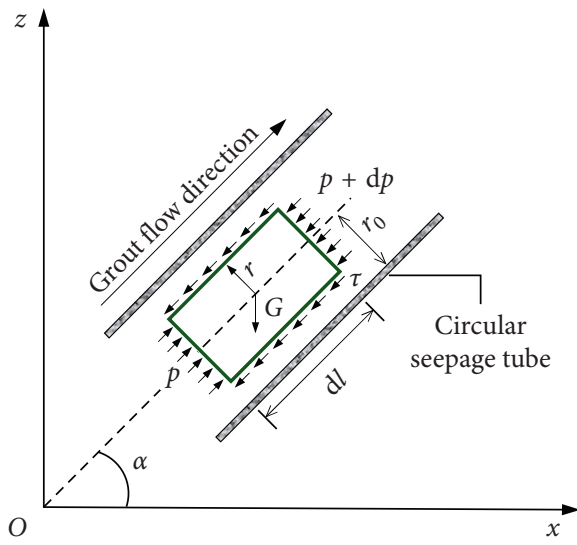

(a)

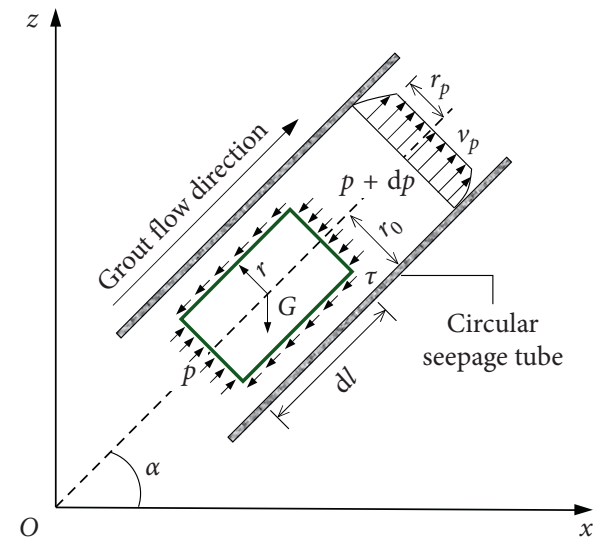

(b)

Figure 2: Geometry of the physical model. (a) Power-law grout and (b) Bingham grout.

\subsection{Mathematical Model Development}

2.2.1. Power-Law Grout. The mechanical equilibrium of the grout microelement for different angles of $\alpha$ is written mathematically as

$$
p \pi r^{2}-(p+\mathrm{d} p) \pi r^{2}-2 \pi r \tau \mathrm{d} l-\rho g \sin \alpha \pi r^{2} \mathrm{~d} l=0,
$$

where $p$ is the injection pressure; $\tau$ is the shear stress on surface of the grout column; $\rho$ is the grout density; and $g$ is the gravitational acceleration.

Equation (1) is simplified into the following equation:

$$
\tau=-\frac{r}{2}\left(\frac{\mathrm{d} p}{\mathrm{~d} l}+\rho g \sin \alpha\right) \text {. }
$$

The rheological equation for power-law fluid is defined as follows [43]:

$$
\tau=C \gamma^{n}=C\left(-\frac{\mathrm{d} v}{\mathrm{~d} r}\right)^{n},
$$

where $C$ is the viscosity coefficient; $\gamma$ is the shear rate; and $n$ is the rheological index.

Together with equations (2) and (3), it yields

$$
\frac{\mathrm{d} v}{\mathrm{~d} r}=-\left(\frac{\tau}{C}\right)^{1 / n}=-\left[-\frac{1}{2 C}\left(\frac{\mathrm{d} p}{\mathrm{~d} l}+\rho g \sin \alpha\right)\right]^{1 / n} r^{1 / n} .
$$
yields

Then, the flow velocity $v$ at any position of the grout fluid

$$
v=\left\{\left[-\frac{1}{2 C}\left(\frac{\mathrm{d} p}{\mathrm{~d} l}+\rho g \sin \alpha\right)\right]^{1 / n} \frac{n}{n+1}\right\}\left(r_{0}^{n+1 / n}-r^{n+1 / n}\right) .
$$

Thus, the grout quantity $q$ per unit time is given as

$$
q=\int_{0}^{r_{0}} 2 \pi r v \mathrm{~d} r=\frac{n \pi}{3 n+1}\left[-\frac{1}{2 C}\left(\frac{\mathrm{d} p}{\mathrm{~d} l}+\rho g \sin \alpha\right)\right]^{1 / n} r_{0}^{3 n+1 / n} .
$$

The average flow velocity $\bar{v}$ can be given as

$$
\bar{v}=\frac{q}{\pi r_{0}^{2}}=\frac{n}{3 n+1}\left[-\frac{1}{2 C}\left(\frac{\mathrm{d} p}{\mathrm{~d} l}+\rho g \sin \alpha\right)\right]^{1 / n} r_{0}^{n+1 / n} .
$$

Solving the Dupuit-Forchheimer equation that defining flow velocity $V=\phi \bar{v}$ [43], it yields 


$$
V=\frac{n \phi}{3 n+1}\left[-\frac{1}{2 C}\left(\frac{\mathrm{d} p}{\mathrm{~d} l}+\rho g \sin \alpha\right)\right]^{1 / n} r_{0}^{n+1 / n}
$$

where $\phi$ is the porosity of porous media.

Here, introducing the effective permeability $K_{e}$ and effective viscosity $\mu_{e}$, which are defined as [43]

$$
\begin{aligned}
K_{e} & =\frac{\phi r_{0}^{2}}{2}\left(\frac{n}{3 n+1}\right), \\
\mu_{e} & =C\left(\frac{3 n+1}{\phi r_{0} n}\right)^{n-1},
\end{aligned}
$$

and equation (8) is rearranged into

$$
V=\left(\frac{K_{e}}{\mu_{e}}\right)^{1 / n}\left[-\left(\frac{\mathrm{d} p}{\mathrm{~d} l}+\rho g \sin \alpha\right)\right]^{1 / n} .
$$

For a representative sphere case, its total surface area is $A=4 \pi l^{2}$. Thus, the total grout quantity $Q$ is given as

$$
Q=V A t
$$

where $t$ is the grouting time.

Equation (11) is rearranged into

$$
V=\frac{Q}{4 \pi t l^{2}}=\left(\frac{K_{e}}{\mu_{e}}\right)^{1 / n}\left[-\left(\frac{\mathrm{d} p}{\mathrm{~d} l}+\rho g \sin \alpha\right)\right]^{1 / n} .
$$

Separating variables from equation (12) yields

$$
p=-\left(\frac{Q}{4 \pi t}\right)^{n} \frac{\mu_{e}}{K_{e}(1-2 n)} l^{1-2 n}-(\rho g \sin \alpha) l+B,
$$

with the boundary conditions $p=p_{0}$ and $l=l_{0}$ and yields

$$
p_{0}=-\left(\frac{Q}{4 \pi t}\right)^{n} \frac{\mu_{e}}{K_{e}(1-2 n)} l^{1-2 n}-(\rho g \sin \alpha) l_{0}+B,
$$

where $p_{0}$ is the injection pressure and $l_{0}$ is the radius of the grouting pipe.

Solving equation (14) yields

$$
B=p_{0}+\left(\frac{Q}{4 \pi t}\right)^{n} \frac{\mu_{e}}{K_{e}(1-2 n)} l^{1-2 n}+(\rho g \sin \alpha) l_{0} .
$$

Together with equations (13) and (15), it yields

$p=p_{0}+\left(\frac{Q}{4 \pi t}\right)^{n} \frac{\mu_{e}}{K_{e}(1-2 n)}\left(l_{0}^{1-2 n}-l^{1-2 n}\right)+\rho g \sin \alpha\left(l_{0}-l\right)$.

Thus, the total grout quantity can be expressed as

$$
Q=\frac{4}{3} \pi l^{3} \phi
$$

Together with equations (16) and (17), it leads to

$$
p=p_{0}+\left(\frac{\phi}{3 t}\right)^{n} \frac{\mu_{e}}{K_{e}(1-2 n)}\left(l_{0}^{1-2 n} l^{3 n}-l^{1+n}\right)+\rho g \sin \alpha\left(l_{0}-l\right) \text {. }
$$

2.2.2. Bingham Grout. In this case, substituting equation (2) into the rheological equation for Bingham grout that is defined as $\tau=\tau_{0}+\mu(-\mathrm{d} v / \mathrm{d} r)$ [43] yields

$$
\frac{\mathrm{d} v}{\mathrm{~d} r}=\frac{\tau_{0}-\tau}{\mu}=\frac{1}{\mu}\left[\tau_{0}+\frac{r}{2}\left(\frac{\mathrm{d} p}{\mathrm{~d} l}+\rho g \sin \alpha\right)\right],
$$

where $\tau_{0}$ is the yield stress.

When $-(r / 2)(\mathrm{d} p / \mathrm{d} l+\rho g \sin \alpha) \leq \tau_{0}$, the grout is not subject to shear action and there exists a flow core with a radius of $r_{p}$ in the tube; when $0 \leq r \leq r_{p}$, no relative motion exists in the grout fluid, and when $r_{p}<r \leq r_{0}$, the relative motion in the fluid produce shear action. Thus, the radius of flow core can be given as

$$
r_{p}=-\frac{2 \tau_{0}}{((\mathrm{~d} p / \mathrm{d} l)+\rho g \sin \alpha)} .
$$

Integrating equation (19) yields

$$
\begin{cases}v=-\frac{1}{\mu}\left[\frac{1}{4}\left(\frac{\mathrm{d} p}{\mathrm{~d} l}+\rho g \sin \alpha\right)\left(r_{0}^{2}-r^{2}\right)+\tau_{0}\left(r_{0}-r\right)\right], & r_{p} \leq r \leq r_{0} \\ v=-\frac{1}{\mu}\left[\frac{1}{4}\left(\frac{\mathrm{d} p}{\mathrm{~d} l}+\rho g \sin \alpha\right)\left(r_{0}^{2}-r_{p}^{2}\right)+\tau_{0}\left(r_{0}-r_{p}\right)\right], & 0 \leq r \leq r_{p} .\end{cases}
$$

The grout quantity per unit time in the circular tube is given by

$$
q=\int_{r_{p}}^{r_{0}} 2 \pi r v d r+\pi r_{p}^{2} v_{p}
$$

Equations (21) and (22) lead to the following equation:

$$
\begin{aligned}
q= & -\frac{\pi r_{0}^{4}}{8 \mu}\left(\frac{\mathrm{d} p}{\mathrm{~d} l}+\rho g \sin \alpha\right)\left[1-\frac{4}{3}\left(\frac{-2 \tau_{0} / r_{0}}{(\mathrm{~d} p / \mathrm{d} l)+\rho g \sin \alpha}\right)\right. \\
& \left.+\frac{1}{3}\left(\frac{-2 \tau_{0} / r_{0}}{(\mathrm{~d} p / \mathrm{d} l)+\rho g \sin \alpha}\right)^{4}\right] .
\end{aligned}
$$

The average velocity of the grout in the tube can be expressed as follows: 


$$
\begin{aligned}
\bar{v}=\frac{q}{\pi r_{0}^{2}}= & \left(\frac{\mathrm{d} p}{\mathrm{~d} l}+\rho g \sin \alpha\right)\left[1-\frac{4}{3}\left(\frac{-2 \tau_{0} / r_{0}}{(\mathrm{~d} p / \mathrm{d} l)+\rho g \sin \alpha}\right)\right. \\
& \left.+\frac{1}{3}\left(\frac{-2 \tau_{0} / r_{0}}{(\mathrm{~d} p / \mathrm{d} l)+\rho g \sin \alpha}\right)^{4}\right] .
\end{aligned}
$$

When there is no grout flow in the tube, it leads to

$$
\frac{-2 \tau_{0} / r_{0}}{(\mathrm{~d} p / \mathrm{d} l)+\rho g \sin \alpha}=1
$$

Thus, the starting pressure gradient $\lambda$ is

$$
\lambda=-\frac{\mathrm{d} p}{\mathrm{~d} l}=\frac{2 \tau_{0}}{r_{0}}+\rho g \sin \alpha .
$$

Here, introducing the variable $K$ is defined as

$$
K=\frac{\phi r_{0}^{2}}{8} .
$$

Thus, the grout flow velocity $V$ is

$$
\begin{aligned}
V= & -\frac{K}{\mu}\left(\frac{\mathrm{d} p}{\mathrm{~d} l}+\rho g \sin \alpha\right)\left[1-\frac{4}{3}\left(\frac{\rho g \sin \alpha-\lambda}{(\mathrm{d} p / \mathrm{d} l)+\rho g \sin \alpha}\right)\right. \\
& \left.+\frac{1}{3}\left(\frac{\rho g \sin \alpha-\lambda}{(\mathrm{d} p / \mathrm{d} l)+\rho g \sin \alpha}\right)^{4}\right] .
\end{aligned}
$$

Together with equations (11) and (28), it yields

$$
\begin{aligned}
V=\frac{Q}{4 \pi l^{2} t} & =-\frac{K}{\mu}\left(\frac{\mathrm{d} p}{\mathrm{~d} l}+\rho g \sin \alpha\right)\left[1-\frac{4}{3}\left(\frac{\rho g \sin \alpha-\lambda}{(\mathrm{d} p / \mathrm{d} l)+\rho g \sin \alpha}\right)\right. \\
& \left.+\frac{1}{3}\left(\frac{\rho g \sin \alpha-\lambda}{(\mathrm{d} p / \mathrm{d} l)+\rho g \sin \alpha}\right)^{4}\right] .
\end{aligned}
$$

Considering the pressure gradient $(-\mathrm{d} p / \mathrm{d} l) \gg \lambda$, equation (29) is simplified as

$$
V=\frac{\mathrm{Q}}{4 \pi l^{2} t}=-\frac{K}{\mu}\left(\frac{\mathrm{d} p}{\mathrm{~d} l}+\rho g \sin \alpha\right)\left[1-\frac{4}{3}\left(\frac{\rho g \sin \alpha-\lambda}{(\mathrm{d} p / \mathrm{d} l)+\rho g \sin \alpha}\right)\right] .
$$

Separating variables and integrating equation (30) yield

$$
p=\frac{Q \mu}{4 \pi t K l t}+\left(\frac{1}{3} \rho g \sin \alpha-\frac{4}{3} \lambda\right) l+C,
$$

with the boundary conditions $p=p_{0}$ and $l=l_{0}$, and equation (31) leads to

$$
\begin{gathered}
p_{0}=\frac{Q \mu}{4 \pi t K l_{0} t}+\left(\frac{1}{3} \rho g \sin \alpha-\frac{4}{3} \lambda\right) l_{0}+C, \\
C=p_{0}-\frac{Q \mu}{4 \pi t K l_{0} t}+\left(\frac{1}{3} \rho g \sin \alpha-\frac{4}{3} \lambda\right) l_{0} .
\end{gathered}
$$

Together with equations (17), (31), and (33), the injection pressure $p$ at any position is given as

$$
p=p_{0}-\frac{\phi \mu}{3 t K l_{0}} l^{3}+\frac{\phi \mu}{3 t K} l^{2}+\left(\frac{4}{3} \lambda-\frac{1}{3} \rho g \sin \alpha\right)\left(l_{0}-l\right) .
$$

2.2.3. Newtonian Grout. Considering the constitutive equation, the Newtonian grout can be regarded as a special form of Bingham grout when $\tau_{0}$ is zero, so the starting pressure gradient $\lambda$ is given as

$$
\lambda=\rho g \sin \alpha .
$$

Then, the injection pressure of Newtonian grout is

$$
p=p_{0}-\frac{\phi \mu}{3 t K l_{0}} l^{3}+\frac{\phi \mu}{3 t K} l^{2}+\rho g \sin \alpha\left(l_{0}-l\right) \text {. }
$$

2.3. Prediction Model of Grout Concretion Dimension. Considering the Bingham and Newtonian grouts are the most commonly used types for grouting practices, thereby the Bingham grout was chosen to establish the prediction model for grout concretion dimension in this section.

Together with equation (34) and boundary conditions $p=0$ and $l=l_{\max }$, which yield

$$
p_{0}-\frac{\phi \mu}{3 t K l_{0}} l_{\max }^{3}+\frac{\phi \mu}{3 t K} l_{\max }^{3}+\left(\frac{4}{3} \lambda-\frac{1}{3} \rho g \sin \alpha\right)\left(l_{0}-l_{\max }\right)=0,
$$

where $l_{\max }$ is the maximum diffusion radius.

When $\alpha$ is $0, \pi / 2$, and $3 \pi / 2$, respectively, the diffusion radius is $l_{\max , 0}, l_{\max , \pi / 2}$, and $l_{\max , 3 \pi / 2}$. Thus, the following equation can be obtained:

$$
\begin{gathered}
p_{0}-\frac{\phi \mu}{3 t K l_{0}} l_{\text {max }, 0}^{3}+\frac{\phi \mu}{3 t K} l_{\max , 0}^{3} \\
+\frac{4}{3} \lambda\left(l_{0}-l_{\max , 0}\right)=0, \\
p_{0}-\frac{\phi \mu}{3 t K l_{0}} l_{\text {max }, \pi / 2}^{3}+\frac{\phi \mu}{3 t K} l_{\max , \pi / 2}^{3} \\
+\left(\frac{4}{3} \lambda-\frac{1}{3} \rho g\right)\left(l_{0}-l_{\max , \pi / 2}\right)=0, \\
p_{0}-\frac{\phi \mu}{3 t K l_{0}} l_{\max , 3 \pi / 2}^{3}+\frac{\phi \mu}{3 t K} l_{\max , 3 \pi / 2}^{3} \\
+\left(\frac{4}{3} \lambda+\frac{1}{3} \rho g\right)\left(l_{0}-l_{\max , 3 \pi / 2}\right)=0,
\end{gathered}
$$

where $l_{\max , 0}, l_{\max , \pi / 2}$, and $l_{\max , 3 \pi / 2}$ is the maximum diffusion radius at horizontal direction, vertical upward direction, and vertical downward direction, respectively.

$l_{\max , 0}, l_{\max , \pi / 2}$, and $l_{\max , 3 \pi / 2}$ can be obtained by solving equations (38a), (38b), and (38c), and then the grout concretion dimension at different directions are 


$$
\begin{aligned}
& D_{x}=D_{y}=2 l_{\text {max }, 0}, \\
& D_{z}=l_{\text {max }, \pi / 2}+l_{\max , 3 \pi / 2},
\end{aligned}
$$

where $D_{x}, D_{y}$, and $D_{z}$ indicated in Figure 1 are the dimensions of grout concretion at transverse direction, longitudinal direction, and vertical direction, respectively.

2.4. Analysis of the Injection Pressure Distribution Law. Some findings of the previous study by Ruan [44] have been utilized to explore the spatial distribution law of injection pressure with diffusion radius variations. In this study, the cement grout was identified as three flow patterns, i.e., power law $((w / c)$ ratio $=0.5-0.7)$, Bingham $((w / c)$ ratio $=0.8-1.0)$, and Newtonian grouts $((w / c)=2.0-10.0)$ based on the variations of the $(w / c)$ ratios. The cement grout with the $(w / c)$ ratios of $0.5,1.0$, and 2.0 together with the aforementioned established models have been used to discuss the relationship between diffusion radius at different directions under different injection pressures. The grout parameters are listed in Table 1, and Figure 3 presents the experimental results.

Figure 3(a) indicates that injection pressure decreases linearly with increase of diffusion radius. Also, attenuation rates of injection pressure at different directions vary marginally in terms of a smaller rheological coefficient. From Figures 3(b) and 3(c), variations of injection pressure along with diffusion radius of Bingham and Newtonian grouts significantly differ from the power-law grout. Nonlinear decreases of injection pressure are found in both two grouts with the increase of diffusion radius. Particularly, compared with the Bingham grout, marginal attenuation in injection pressure was observed within $10 \mathrm{~cm}$ of the radius for Newtonian grout, while the slight difference emerges when radius is beyond $10 \mathrm{~cm}$. The injection pressure distributes diminishingly at directions of $3 \pi / 2,0$, and $\pi / 2$ in terms of the same diffusion radius.

2.5. Comparison of Diffusion Radius with and without considering Self-Gravity Effect. The proposed models were further used to discuss diffusion radius in aspects of considering and not considering self-gravity effects of the three grouts, and results are shown in Figure 4.

It can be seen from Figure 4 that three grouts diffuse approximately in an "ellipsoidal" shape. Though values of the diffusion radius considering gravity effects in horizontal direction exhibit little or even no differences compared with not considering gravity effects, the remarkable differences of the diffusion radius in vertical direction have proved the necessity to take into account the grout gravity effects. The emergence of gravity leads to the decrease of the diffusion radius in the upper part while that increase in the lower part. The Newtonian grout produces the maximum diffusion radius compared with the other two grouts whether considering the gravity effects or not.

\section{Experimental Campaign}

3.1. Materials. The cement grout used in experiments was made from the ordinary Portland cement P.I 42.5 (commercial cement produced in Shandong Lucheng Cement Company) and water. The manufactured coarse sand with dimension varying from $0.25 \mathrm{~mm}$ to $2.0 \mathrm{~mm}$ was used to produce the injected medium, which is presented in Table 2. The displayed shear strength indexes of the used sand were determined by a series of laboratory triaxial compression tests.

3.2. Experimental Program. The grouting test with three different injection pressures and $(w / c)$ ratios was designed in the experiments. Total 9 groups of grouting tests were conducted, in which the injection pressure is $80 \mathrm{kPa}$, $100 \mathrm{kPa}$, and $120 \mathrm{kPa}$, respectively, while $(w / c)$ ratio is 1.0 , 1.5 , and 2.0, respectively. The detailed information of the designed tests is presented in Table 3 . The whole grouting model test system consists of four parts: the first part is the model box system; the second part is the grouting system (including grout tank, grouting pipe, and grouting pump); the third part is the pressure system (including air compressor and intake pipe); and the fourth part is the measurement system (including electronic scale and pressure gauge), which are presented in Figures 5 and 6. The model box was made of plexiglass with a steel girder as the support member, and the geometric length, width, and height of the box are $70 \mathrm{~cm}, 45 \mathrm{~cm}$, and $65 \mathrm{~cm}$, respectively. During the grouting test, the pressure with a certain value that measured by the gauge is generated by the air compressor. The highpressure gas is connected with the grouting pump, and then the grout is forced to flow through the grouting pipe and finally injected into the sand.

\section{Results and Discussion}

4.1. Analysis of the Grout Quantity. In this section, the grout quantity changing with time under different injection pressures and $(w / c)$ ratios is analyzed by recorded results, which are shown in Figure 7.

From Figure 7, it is indicated that the grout quantity sharply increases at the initial phase, then slowly increases, and finally tends to be stable. More time is required to reach a steady state under a larger injection pressure. Differing from the two other ratios, the $(w / c)$ ratio of 1.0 brings about an obvious two-stage change in grout quantity over time. Specifically, grout quantity increases sharply within 0.5 minutes at the first stage, and then remains stable in a certain time. At the second stage, the quantity increases slightly in a short time and eventually tends to be stable. As for the two larger ratios, the grout quantity mainly reaches its maximum in 4 minutes and remains stable. When the $(w / c)$ ratio is set as 1.5 , the grout quantity under an injection pressure of $100 \mathrm{kPa}$ is less than that the quantity under an injection pressure of $80 \mathrm{kPa}$. This may be because compaction process leads to difference between the number and arrangement of pore passages in the soil.

4.2. Analysis of the Grout Concretion Dimension. The diagram for the dimension measurement of grout concretion from grouting test at three directions (i.e., transverse, vertical, 
TABLE 1: Parameters of three grouts used for the study.

\begin{tabular}{lcccccccc}
\hline Grout type & $\mu_{e}(\mathrm{~Pa} \cdot \mathrm{s})$ & $\tau_{0}(\mathrm{~Pa})$ & $\rho\left(\mathrm{g} / \mathrm{cm}^{3}\right)$ & $K_{e}(\mathrm{~cm} / \mathrm{s})$ & $n$ & $l_{0}(\mathrm{~cm})$ & $t(\mathrm{~s})$ & $p(\mathrm{kPa})$ \\
\hline Power law & - & - & 1.86 & 0.03 & 0.4 & 2.5 & 600 & 200 \\
Bingham & 0.0096 & 1.563 & 1.49 & 0.03 & 0.4 & 2.5 & 600 \\
Newtonian & 0.0047 & 0 & 1.30 & 0.03 & 0.4 & 2.5 & 600 \\
\hline
\end{tabular}

Note. $n$ is porosity.

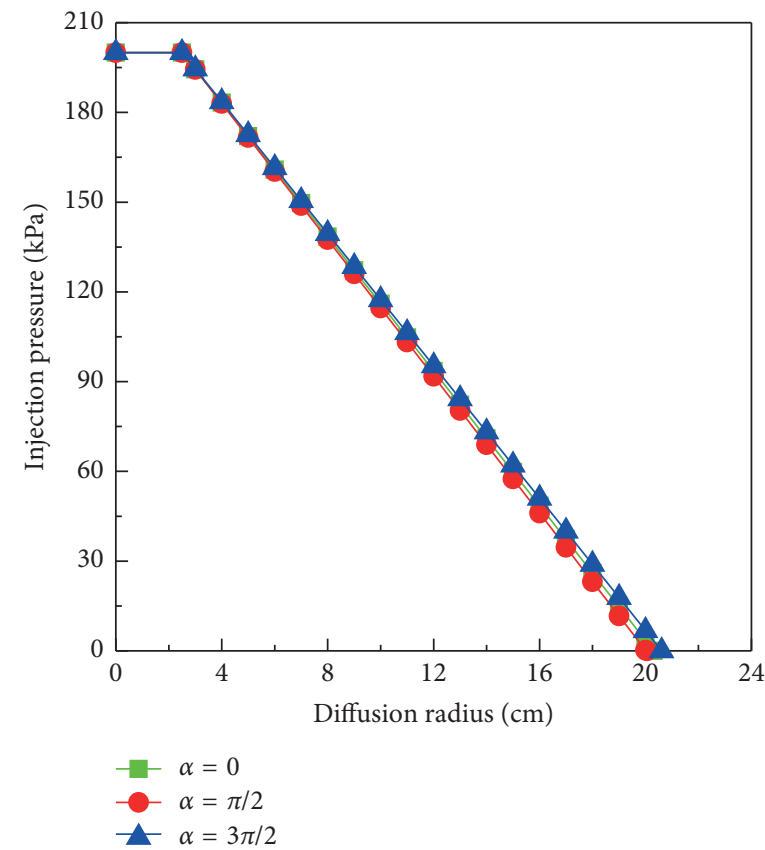

(a)

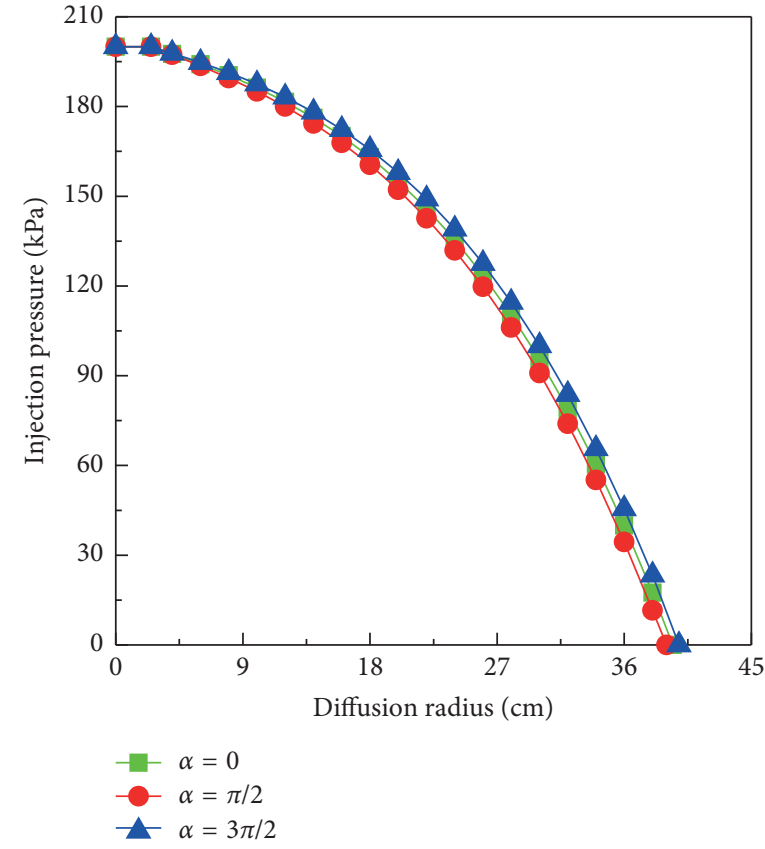

(b)

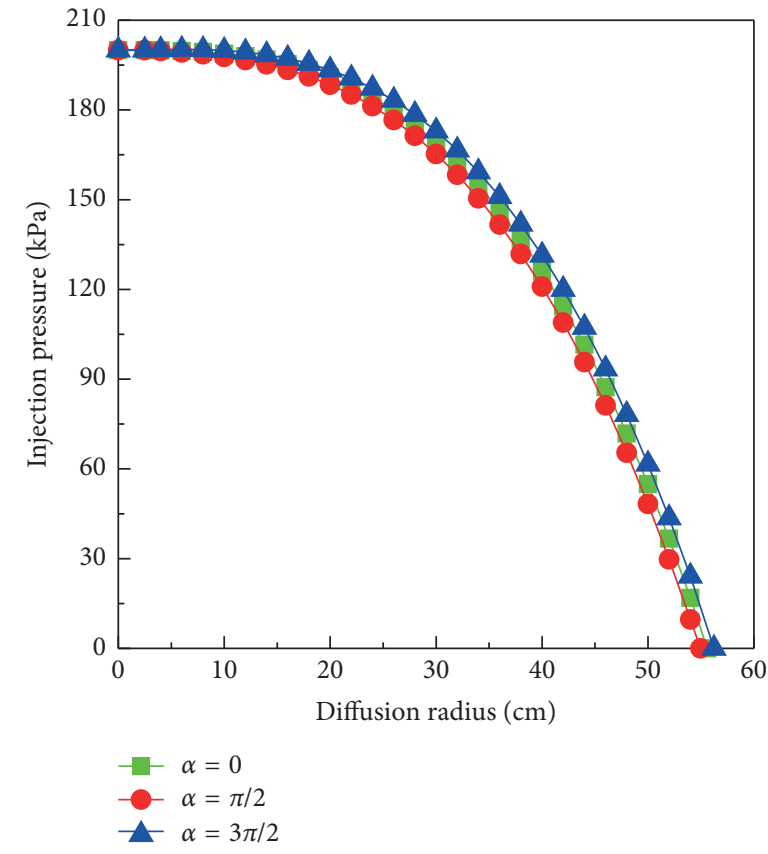

(c)

FIGURE 3: Injection pressure distribution of grouts at different directions. (a) Power-law grout, (b) Bingham grout, and (c) Newtonian grout.

and longitudinal) is shown in Figure 8. Also, the theoretical values obtained based on the dimension prediction model are also used for the comparative analysis and verification.
4.2.1. Transverse Dimension. As detailed in Figure 9, the experimental and theoretical values of the transverse dimension of grout concretion are approximately linearly 


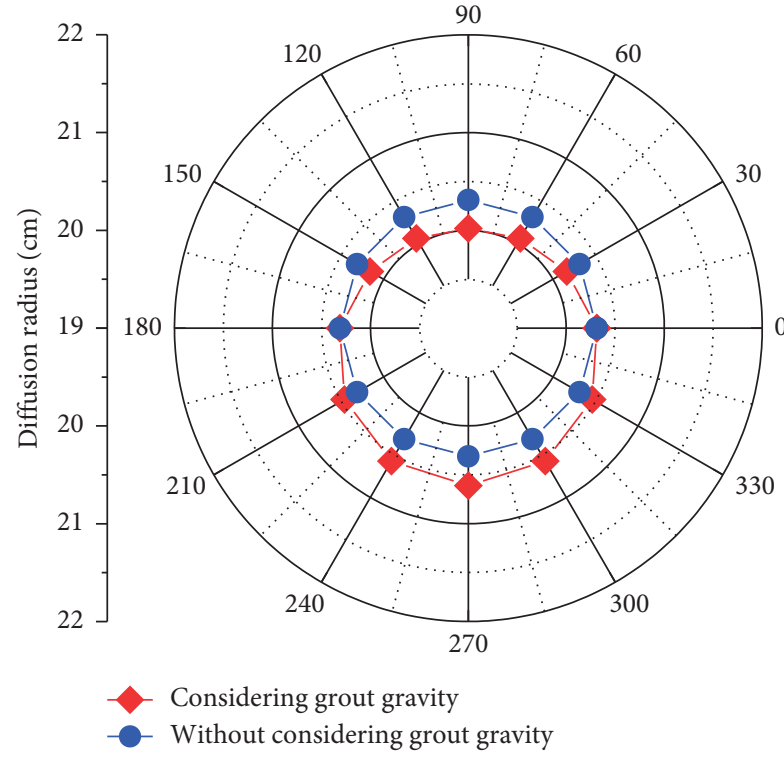

(a)

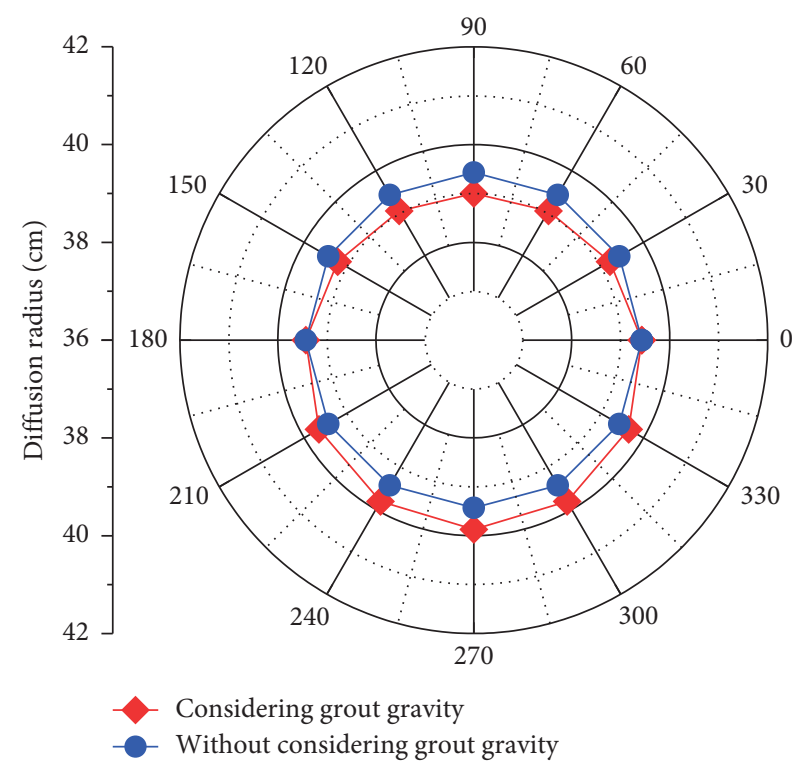

(b)

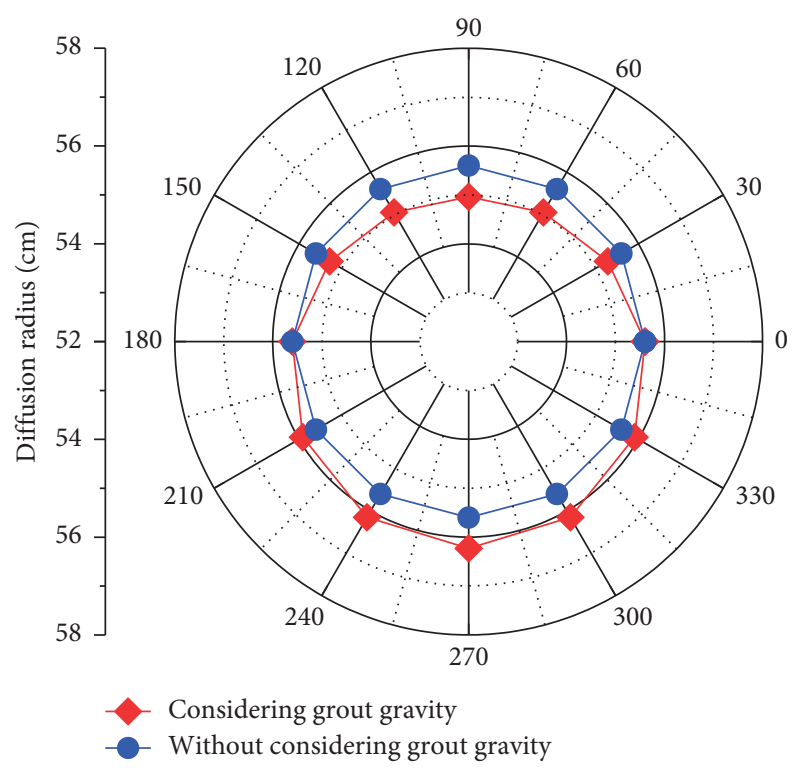

(c)

Figure 4: Diffusion radius of different cement grouts. (a) Power-law grout, (b) Bingham grout, and (c) Newtonian grout.

TABLE 2: Physical and mechanical parameters of the used sand.

\begin{tabular}{lccccccc}
\hline$G_{s}\left(\mathrm{~g} / \mathrm{cm}^{3}\right)$ & $n$ & $\omega(\%)$ & $K_{e}(\mathrm{~cm} / \mathrm{s})$ & $\varphi\left(^{\circ}\right)$ & $c(\mathrm{kPa})$ & $C_{u}$ & $C_{c}$ \\
\hline 2.65 & 0.4 & 4 & 0.0344 & 34.9 & 4 & 2.74 & 1.29 \\
\hline
\end{tabular}

Note. $G_{s}$ is the specific gravity, $n$ is porosity, $\omega$ is water content, $\varphi$ is internal friction angle, $c$ is cohesion, $C_{u}$ is nonuniformity coefficient, and $C_{c}$ is curvature coefficient.

correlated with injection pressure, and the growth rate is roughly the same. The theoretical values at the $(w / c)$ ratios of 1.0, 1.5, and 2.0 are ca. 3.0, 2.0, and 1.5 times, respectively, of the experimental values. It is predicted that the increase of $(w / c)$ ratio can reduce difference between the experimental and theoretical values and finally obtain more reliable results.
4.2.2. Vertical Dimension. The vertical dimension of grout concretion changing with the injection pressure is shown in Figure 10. The results suggest that vertical dimension presents a linear correlation with the injection pressure for the theoretical values, while the correlation under the experimental conditions shows nonlinear characteristics. Specifically, experimental transverse dimension at a ratio of 
TABLE 3: Material property of the used cement grouts.

\begin{tabular}{lccccccccc}
\hline No. & $(w / c)$ ratio & $p_{0}(\mathrm{kPa})$ & $\mu_{e}(\mathrm{~Pa} \cdot \mathrm{s})$ & $\tau_{0}(\mathrm{~Pa})$ & $\rho\left(\mathrm{g} / \mathrm{cm}^{3}\right)$ & $K_{e}(\mathrm{~cm} / \mathrm{s})$ & $n$ & $l_{0}(\mathrm{~cm})$ & $t(\mathrm{~s})$ \\
\hline 1 & 1.0 & 80 & 0.0096 & 1.563 & 1.49 & 0.0344 & 0.4 & 0.5 \\
2 & 1.0 & 100 & 0.0096 & 1.563 & 1.49 & 0.0344 & 0.4 & 0.5 & 120 \\
3 & 1.0 & 120 & 0.0096 & 1.563 & 1.49 & 0.0344 & 0.4 & 0.5 & 120 \\
4 & 1.5 & 80 & 0.0073 & 0.3568 & 1.37 & 0.0344 & 0.4 & 0.5 & 120 \\
5 & 1.5 & 100 & 0.0073 & 0.3568 & 1.37 & 0.0344 & 0.4 & 0.5 & 120 \\
6 & 1.5 & 120 & 0.0073 & 0.3568 & 1.37 & 0.0344 & 0.4 & 0.5 \\
7 & 2.0 & 80 & 0.0067 & 0.0971 & 1.30 & 0.0344 & 0.4 & 0.5 \\
8 & 2.0 & 100 & 0.0067 & 0.0971 & 1.30 & 0.0344 & 0.4 & 0.5 & 120 \\
9 & 2.0 & 120 & 0.0067 & 0.0971 & 1.30 & 0.0344 & 0.4 & 0.5 \\
\hline
\end{tabular}

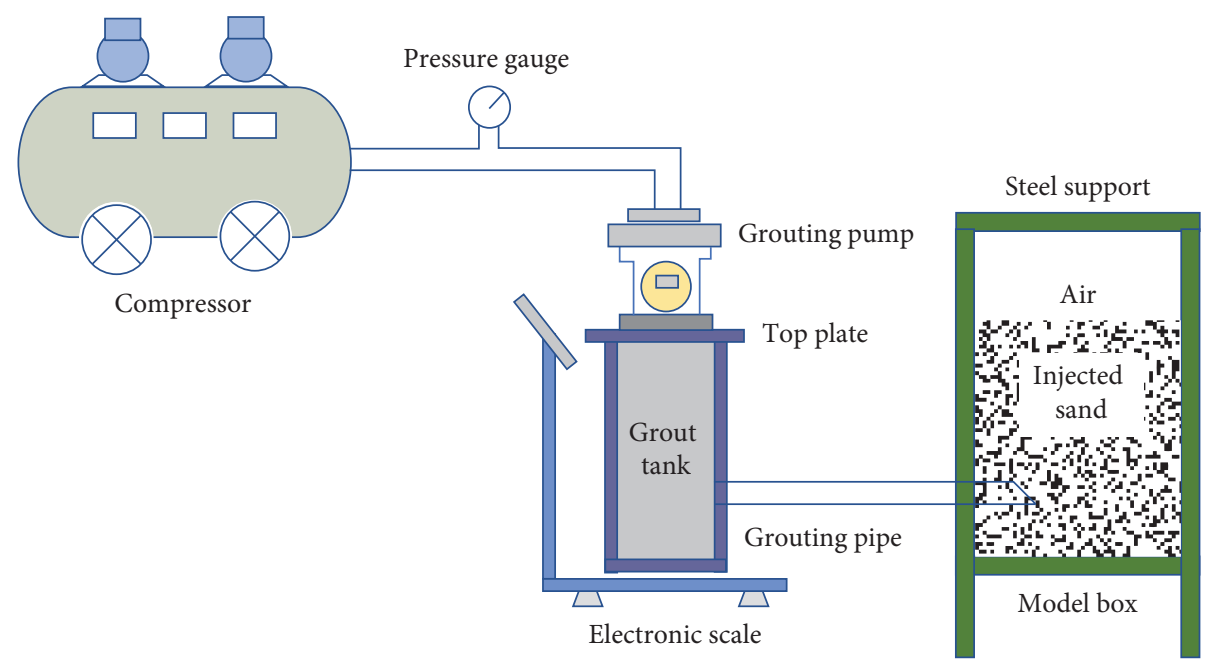

Figure 5: Schematic diagram of the grout injection system.

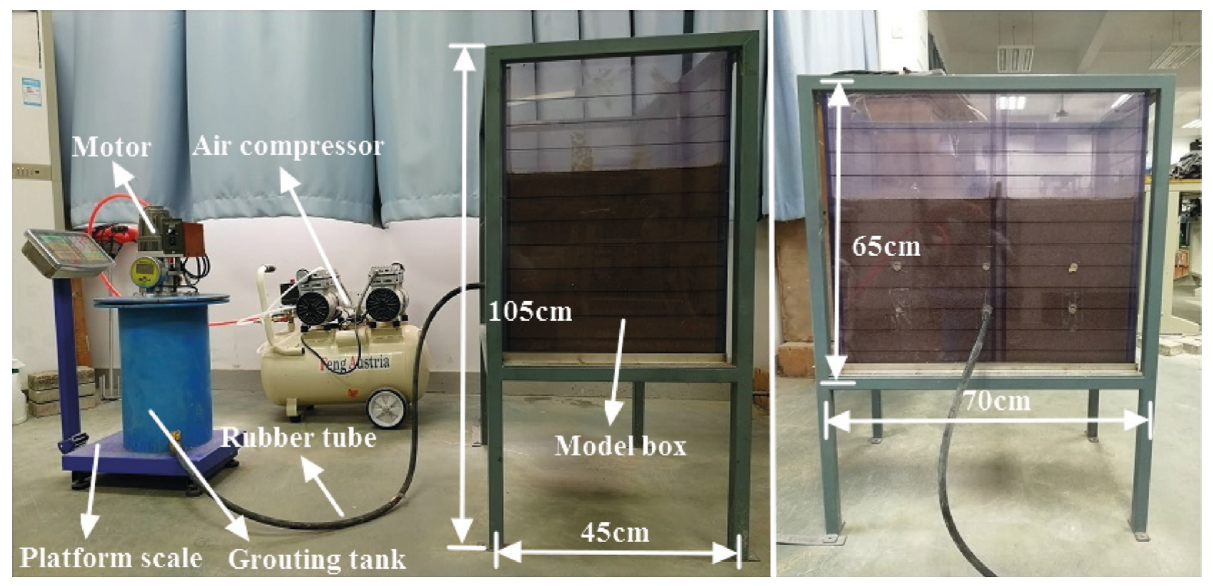

Figure 6: Real map of the test devices.

2.0 decreased slightly when the injection pressure exceeds $100 \mathrm{kPa}$, which verifies the randomness of the grout diffusion at vertical direction. The theoretical values at the $(w / c)$ ratios of 1.0, 1.5, and 2.0 are ca. 3.0, 2.0, and 2 times, respectively, of the experimental values. It appears that the difference between experimental and theoretical values tends to decrease with the increase of $(w / c)$ ratio, but this trend will gradually weaken.
4.2.3. Longitudinal Dimension. The longitudinal dimensions of grout concretion under experimental and theoretical conditions are presented in Figure 11. The data confirm a linear correlation between the longitudinal dimension and injection pressure in both experimental value and theoretical value. It seems that the experimental value of grout concretion at longitudinal direction is gradually close to the theoretical value with the increase of $(w / c)$ ratio, which 


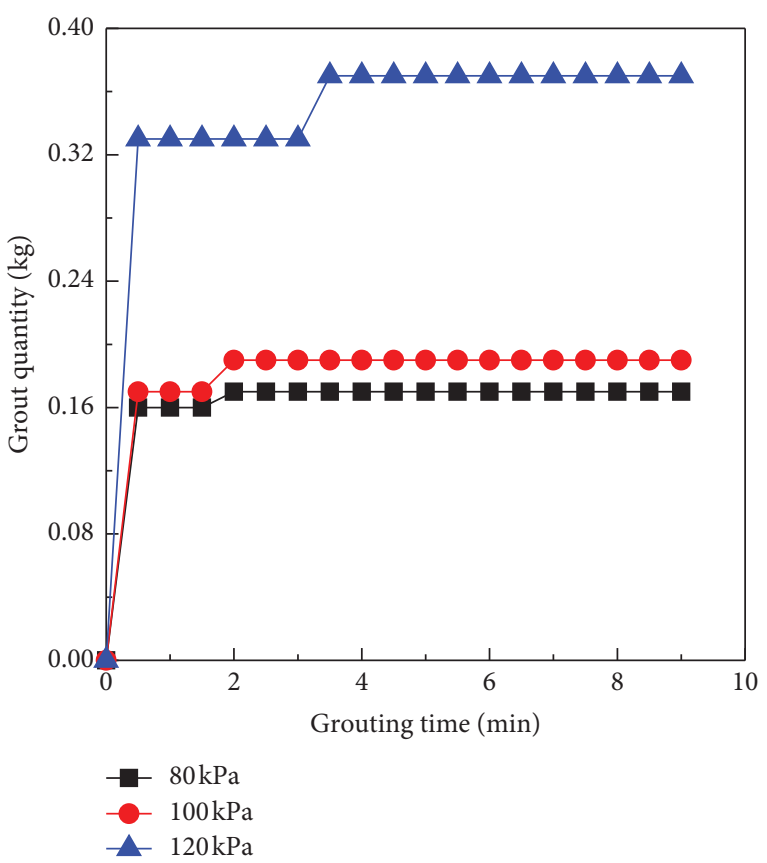

(a)

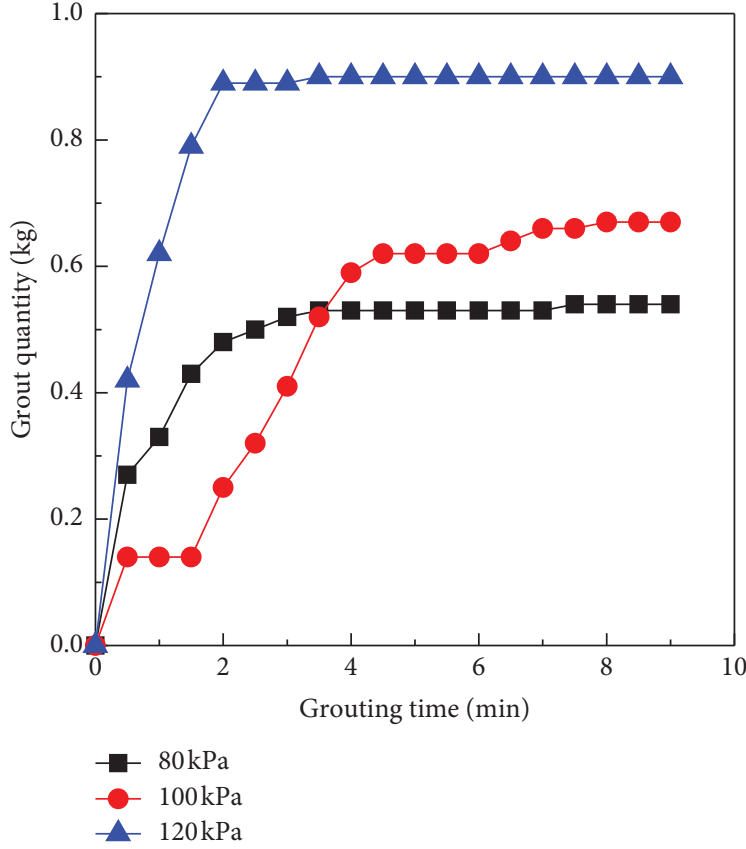

(b)

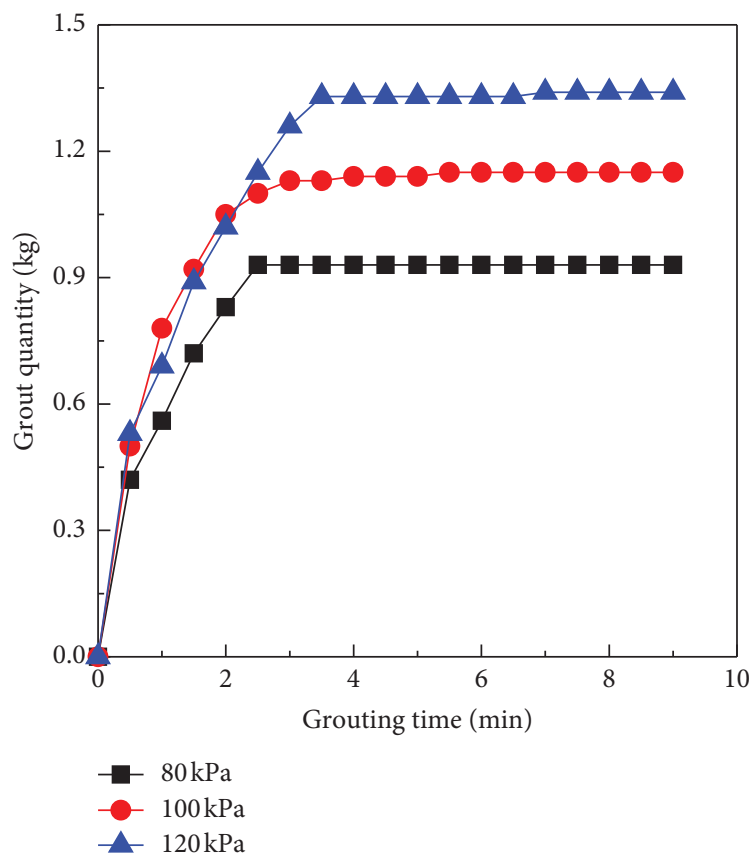

(c)

Figure 7: Grout quantity vs. time under different injection pressures and $(w / c)$ ratios. $(w / c)$ ratios of (a) 1.0, (b) 1.5 , and (c) 2.0 .

indicates that the theoretical model can be effectively used to predict the grout concretion dimension.

It is noted that there is a considerable difference between theoretical and experimental results at the same injection pressure, and this is because of pressure loss, increases of grout viscosity, and filtration effect. Specifically, friction effect caused by grout flowing in the grouting pipe results in pressure loss. The insufficient tightness of the pipe joint also leads to a loss of injection pressure. The viscosity of cement- based grout is featured by the time-varying property. During cement hydration, the viscosity and viscous resistance of grout increase over time, causing an adverse impact on grout diffusion [41]. When grout is injected into the soil, part of cement particles is blocked by skeleton of soil particles, resulting in stagnation and filtration of these cement particles as well as decrease of grout concentration and blockage of the soil pores. The grouting effect is significantly influenced by limited diffusion channels of the grout [28, 45-47]. 


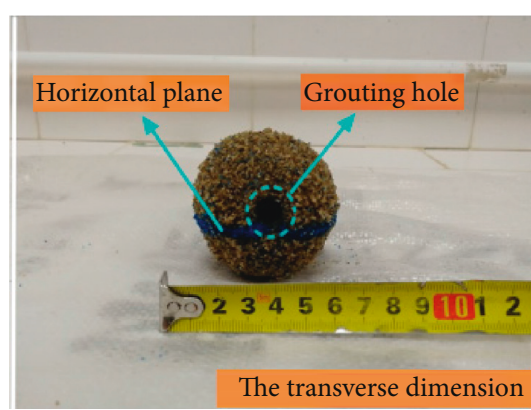

(a)

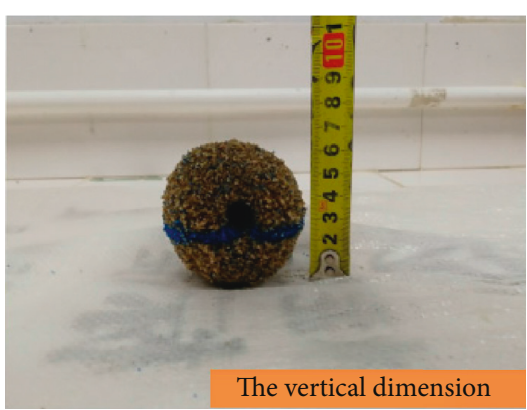

(b)

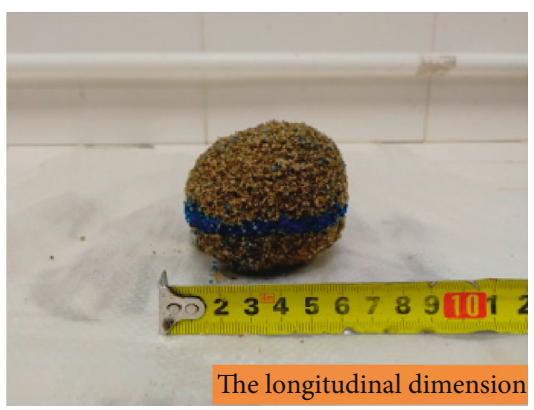

(c)

FIGURE 8: Diagram of the dimension of grout concretion.

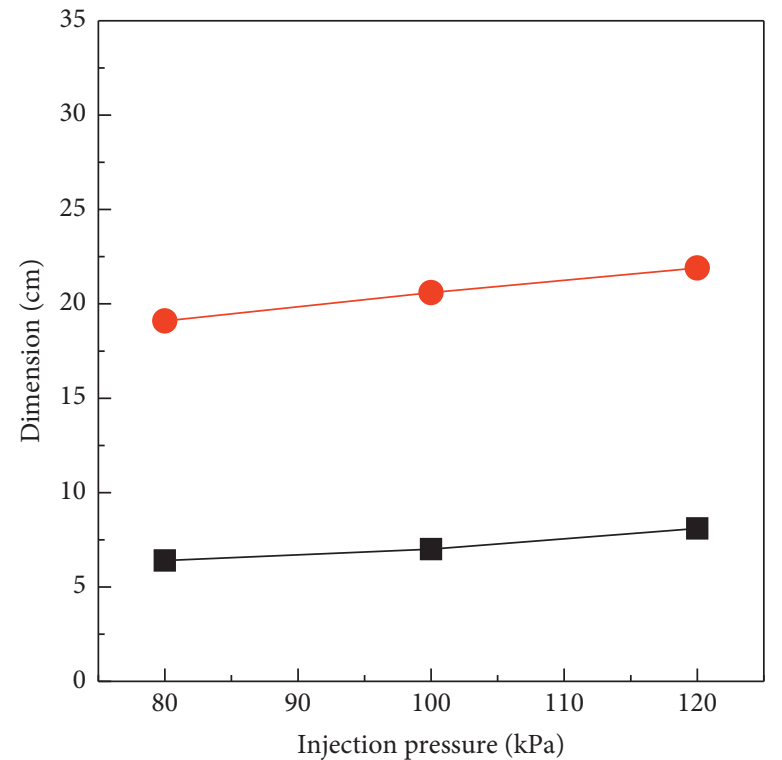

Experimental value

Theoretical value

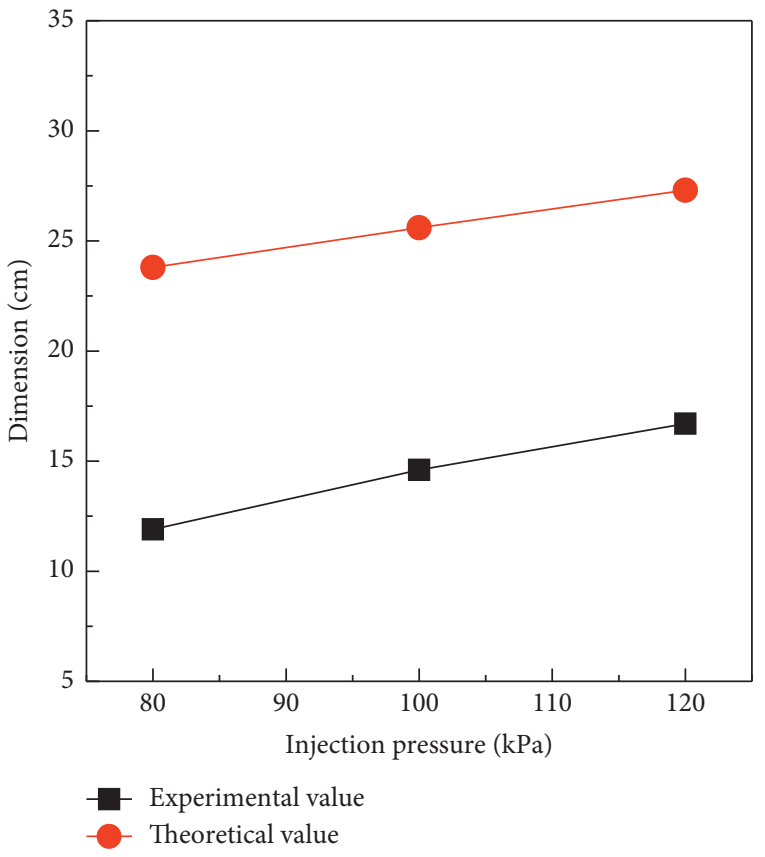

(b)

Figure 9: Continued. 


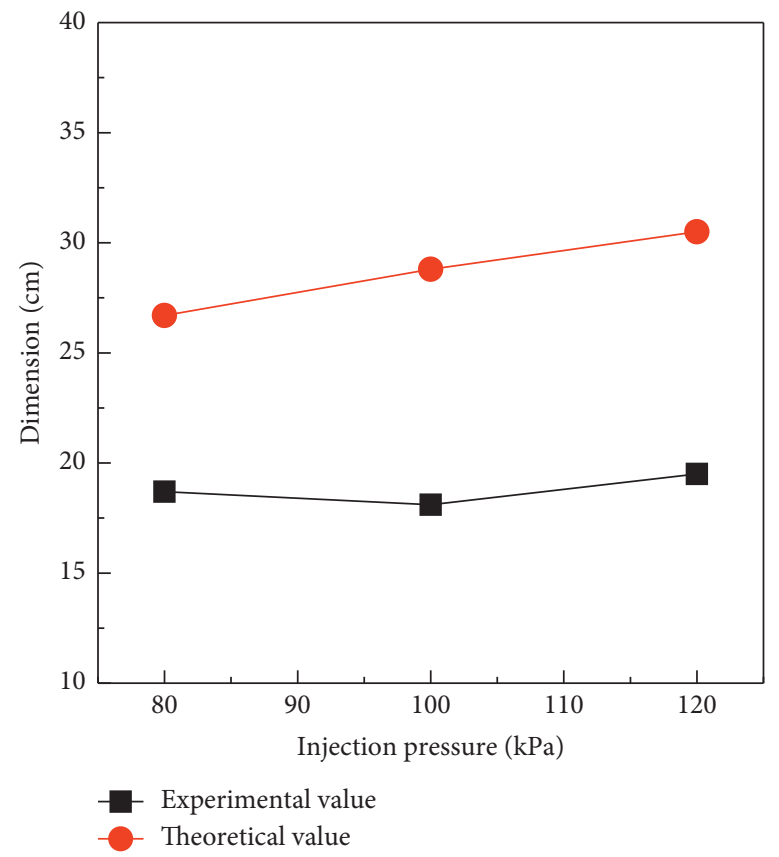

(c)

Figure 9: The transverse dimension of grout concretion under different $(w / c)$ ratios. ( $w / c)$ ratios of (a) 1.0, (b) 1.5, and (c) 2.0.

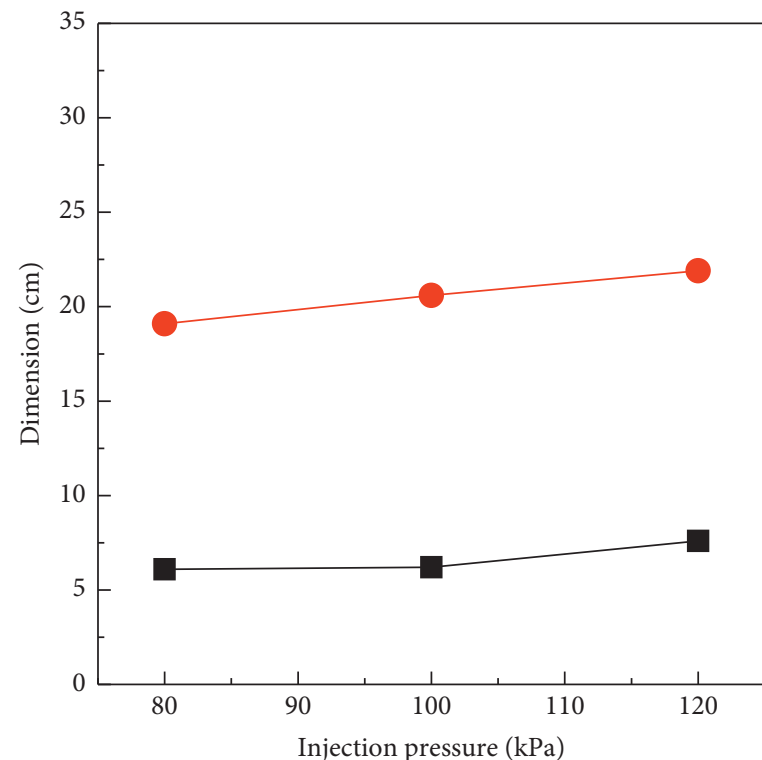

Experimental value

Theoretical value

(a)

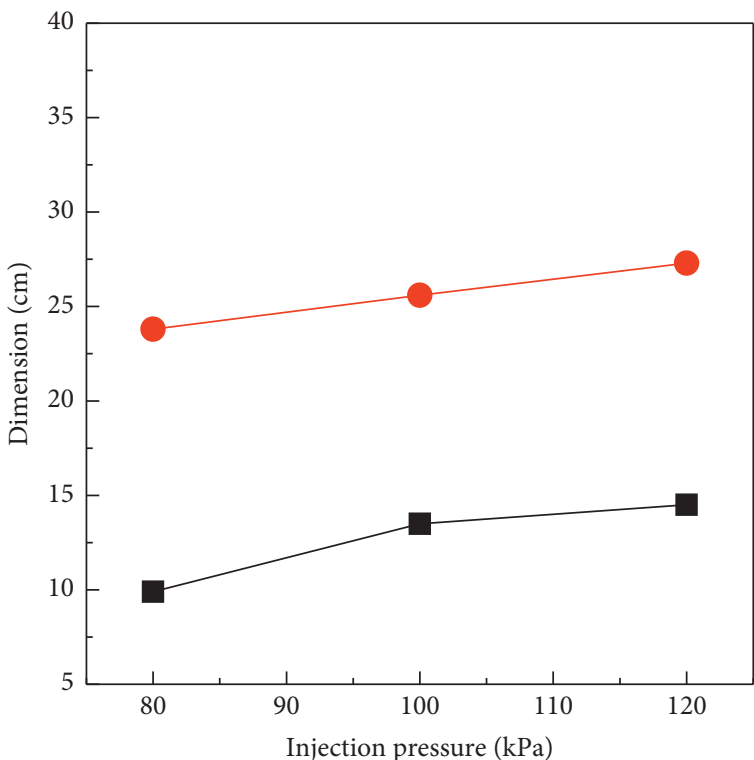

Experimental value Theoretical value

(b)

FIGURE 10: Continued. 


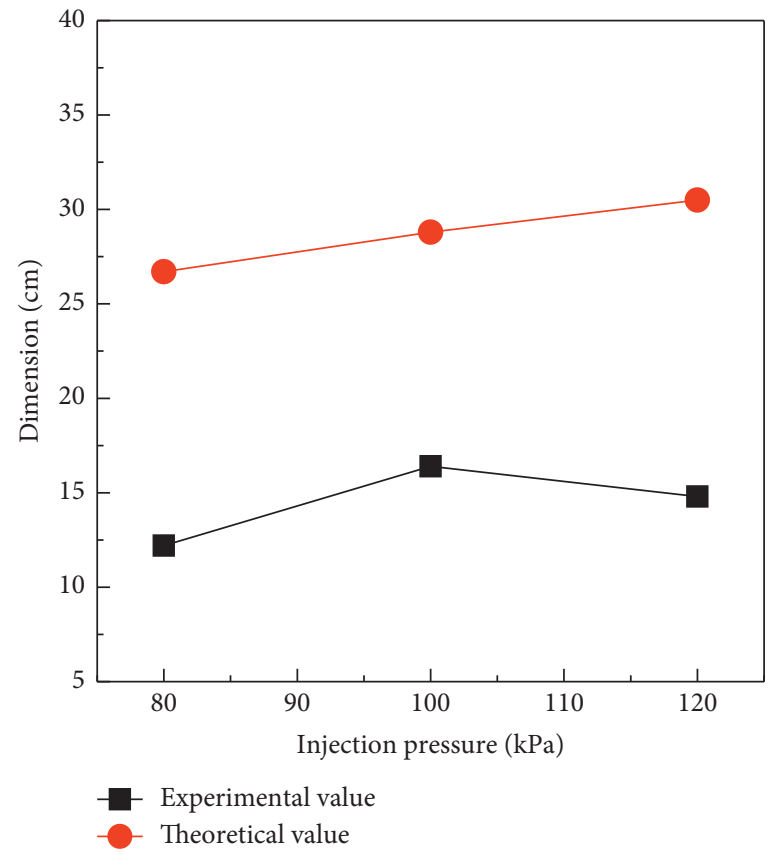

(c)

Figure 10: The vertical dimension of grout concretion under different $(w / c)$ ratios. $(w / c)$ ratios of (a) 1.0, (b) 1.5, and (c) 2.0.

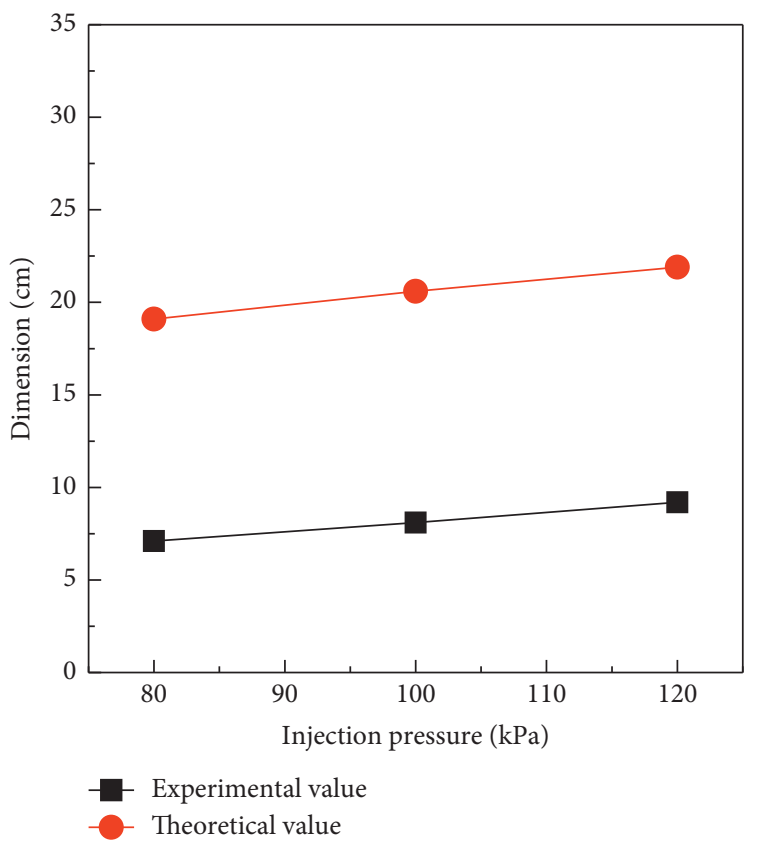

(a)

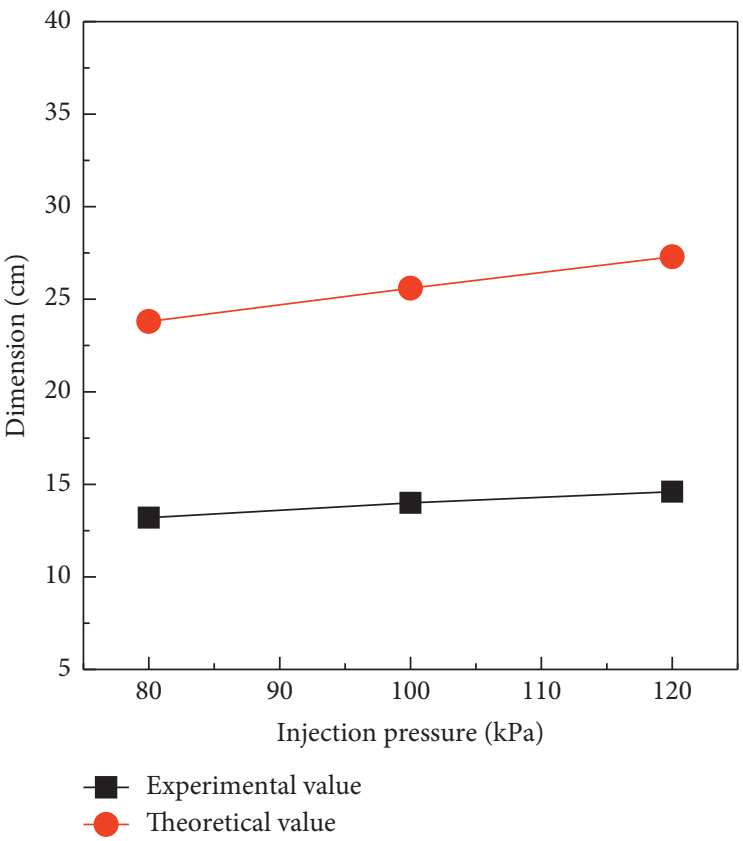

(b)

FIgURE 11: Continued. 


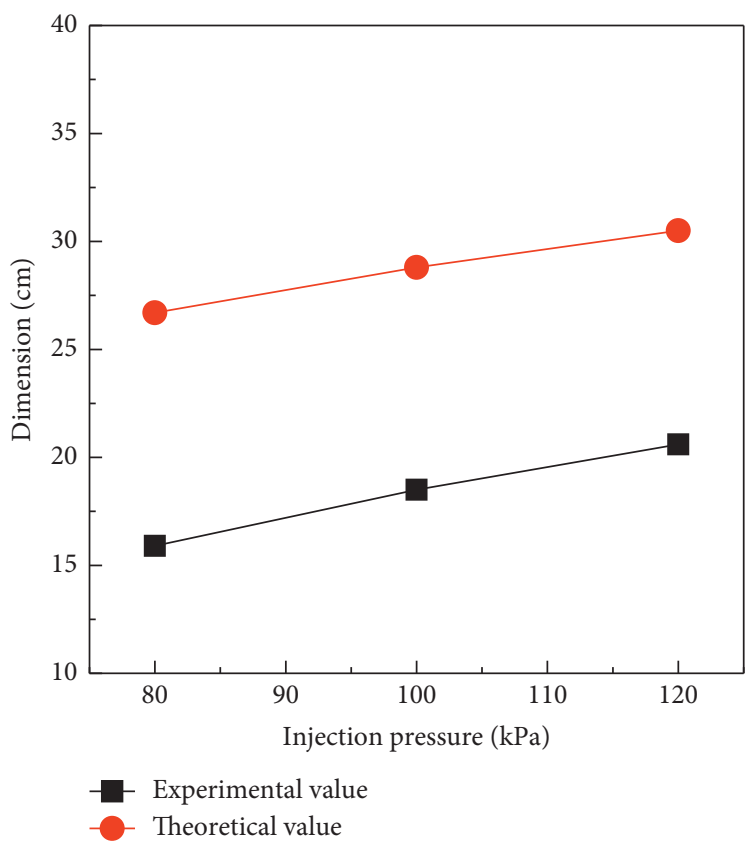

(c)

FIgURE 11: The longitudinal dimension of grout concretion under different $(w / c)$ ratios.

\section{Conclusions}

(1) Mathematical models of permeation grouting for power law, Bingham, and Newtonian grouts are constructed. The self-gravity effect on grout permeation and diffusion has been taken into consideration.

(2) For the power-law cement grout, the injection pressure decreases linearly with increase of diffusion radius, while nonlinear decreases of injection pressure were confirmed in Bingham and Newtonian grouts with the increase of diffusion radius.

(3) Three grouts approximately diffuse in an "ellipsoidal" shape. The grout self-gravity is closely related to the variations of diffusion radius in vertical direction. The Newtonian grout produces the maximum diffusion radius compared with the other two grouts whether the gravity effects were considered or not.

(4) The grout quantity under a smaller $(w / c)$ ratio exhibits significant differences and undergoes two increasing stages, whereas the quantity simply tends to be stable after it reaches its maximum under the two larger $(w / c)$ ratios.

(5) The proposed prediction model for grout concretion dimension shows good agreements with the experimental data, which verify its practicability.

\section{Data Availability}

The data used to support the findings of this study are included within the article.

\section{Conflicts of Interest}

The authors declare no conflicts of interest.

\section{Acknowledgments}

This research was financially supported by the National Natural Science Foundation of China (no. 51678363), Shenzhen Science and Technology Project (no. JCYJ20150525092941052), and Underground Engineering (Tongji University) (no. KLETJGE-B0905).

\section{References}

[1] M. Chang, T.-W. Mao, and R.-C. Huang, "A study on the improvements of geotechnical properties of in-situ soils by grouting," Geomechanics and Engineering, vol. 10, no. 4, pp. 527-546, 2016.

[2] E. N. Bellendir, A. V. Aleksandrov, M. G. Zertsalov, and A. N. Simutin, "Building and structure protection and leveling using compensation grouting technology," Power Technology and Engineering, vol. 50, no. 2, pp. 142-146, 2016.

[3] J. L. Qiu, H. Q. Liu, J. X. Lai, H. P. Lai, J. X. Chen, and K. Wang, "Investigating the long-term settlement of a tunnel built over improved loessial foundation soil using jet grouting technique," Journal of Performance of Constructed Facilities, vol. 32, no. 5, Article ID 04018066, 2018.

[4] X. L. Wang, J. X. Lai, R. S. Garnes, and Y. B. Luo, "Support system for tunnelling in squeezing ground of Qingling-Daba mountainous area: a case study from soft rock tunnels," Advances in Civil Engineering, vol. 2019, Article ID 8682535, 17 pages, 2019.

[5] X. X. Nie, S. S. Feng, S. D. Zhang, and Q. Gan, "Simulation study on the dynamic ventilation control of single head roadway in high- altitude mine based on thermal comfort," Advances in Civil Engineering, vol. 2019, Article ID 2973504, 12 pages, 2019.

[6] S. B. Zhang, S. Y. He, J. L. Qiu, W. Xu, R. S. Garnes, and L. X. Wang, "Displacement characteristics of an urban tunnel 
in silty soil by shallow tunnelling method," Advances in Civil Engineering, vol. 2019, 2019.

[7] N. Shrivastava and K. Zen, "An experimental study of compaction grouting on its densification and confining effects," Geotechnical and Geological Engineering, vol. 36, no. 5, pp. 1-11, 2017.

[8] J. M. Minto, E. Maclachlan, G. El Mountassir, and R. J. Lunn, "Rock fracture grouting with microbially induced carbonate precipitation," Water Resources Research, vol. 52, no. 11, pp. 8827-8844, 2016.

[9] L. Zou, U. Håkansson, and V. Cvetkovic, "Cement grout propagation in two-dimensional fracture networks: impact of structure and hydraulic variability," International Journal of Rock Mechanics and Mining Sciences, vol. 115, pp. 1-10, 2019.

[10] L. Hässler, U. Håkansson, and H. Stille, "Computer-simulated flow of grouts in jointed rock," Tunnelling and Underground Space Technology, vol. 7, no. 4, pp. 441-446, 1992.

[11] L. Zou, U. Håkansson, and V. Cvetkovic, "Two-phase cement grout propagation in homogeneous water-saturated rock fractures," International Journal of Rock Mechanics and Mining Sciences, vol. 106, pp. 243-249, 2018.

[12] D. Park and J. Oh, "Permeation grouting for remediation of dam cores," Engineering Geology, vol. 233, pp. 63-75, 2018.

[13] Y. Zheng, J. Xiong, T. Liu, X. Yue, and J. Qiu, "Performance of a deep excavation in Lanzhou strong permeable sandy gravel strata," Arabian Journal of Geosciences, vol. 12, no. 16, p. 12, 2019.

[14] H. Sun, Q. P. Wang, P. Zhang, Y. J. Zhong, and X. B. Yue, "Spatiotemporal characteristics of tunnel traffic accidents in China from 2001 to present," Advances in Civil Engineering, vol. 2019, Article ID 4536414, 16 pages, 2019.

[15] B.-T. Roque, P.-M. Pablo, C.-F. Daniel, and B.-F. Elena, "Borehole thermal response and thermal resistance of four different grouting materials measured with a TRT," Applied Thermal Engineering, vol. 53, no. 1, pp. 13-20, 2013.

[16] J. Zhang and Y. Sun, "Experimental and mechanism study of a polymer foaming grouting material for reinforcing broken coal mass," KSCE Journal of Civil Engineering, vol. 23, no. 1, pp. 346-355, 2019.

[17] N. Cristelo, E. Soares, I. Rosa et al., "Rheological properties of alkaline activated fly ash used in jet grouting applications," Construction and Building Materials, vol. 48, pp. 925-933, 2013.

[18] C. Ma, Y. H. Tan, E. B. Li, Y. S. Dai, and M. Yang, "Highperformance grouting mortar based on mineral admixtures," Advances in Materials Science and Engineering, vol. 2015, Article ID 425456, 2015.

[19] X. Yue, Y. Xie, H. Zhang, Y. Niu, S. Zhang, and J. Li, "Study on geotechnical characteristics of marine soil at Hong KongZhuhai-Macao tunnel," Marine Georesources \& Geotechnology, vol. 37, no. 8, pp. 1-12, 2019.

[20] S. Y. He, J. X. Lai, L. X. Wang, and K. Wang, "A literature review on properties and applications of grouts for shield tunnel," Construction and Building Materials, vol. 231, pp. 468-482, 2020.

[21] M. Mollamahmutoglu and Y. Yilmaz, "Engineering properties of medium-to-fine sands injected with microfine cement grout," Marine Georesources \& Geotechnology, vol. 29, no. 2, pp. 95-109, 2011.

[22] I. N. Markou and A. I. Droudakis, "Factors affecting engineering properties of microfine cement grouted sands," Geotechnical and Geological Engineering, vol. 31, no. 4, pp. 1041-1058, 2013.

[23] F. Huang, J. G. Lyu, H. Gao, and G. H. Wang, "Modified Maag's spherical diffusion model of vacuum penetration grouting," Mathematical Problems in Engineering, vol. 2018, Article ID 1758651, 7 pages, 2018.

[24] B.-K. Kim, J.-J. Park, Y.-S. Kwon, G.-B. Jeong, and I.-M. Lee, "Groutability enhancement effect of oscillatory injection in cement-based permeation grouting," Geotechnical Testing Journal, vol. 42, no. 1, pp. 64-85, 2019.

[25] F. Bouchelaghem, "Multi-scale modelling of the permeability evolution of fine sands during cement suspension grouting with filtration," Computers and Geotechnics, vol. 36, no. 6, pp. 1058-1071, 2009.

[26] G. Gustafson, J. Claesson, and A. Fransson, "Steering parameters for rock grouting," Journal of Applied Mathematics, vol. 2013, pp. 1-9, 2013.

[27] J. Yoon and C. S. El Mohtar, "A filtration model for evaluating maximum penetration distance of bentonite grout through granular soils," Computers and Geotechnics, vol. 65, pp. 291301, 2015.

[28] S. Maghous, Z. Saada, L. Dormieux, J. Canou, and J. C. Dupla, "A model for in situ grouting with account for particle filtration," Computers and Geotechnics, vol. 34, no. 3, pp. 164174, 2007.

[29] Q.-S. Zhang, L.-Z. Zhang, R.-T. Liu, S.-C. Li, and Q.-Q. Zhang, "Grouting mechanism of quick setting slurry in rock fissure with consideration of viscosity variation with space," Tunnelling and Underground Space Technology, vol. 70, pp. 262-273, 2017.

[30] M. Eriksson, H. Stille, and J. Andersson, "Numerical calculations for prediction of grout spread with account for filtration and varying aperture," Tunnelling and Underground Space Technology, vol. 15, no. 4, pp. 353-364, 2000.

[31] M. J. Yang, Z. Q. Yue, P. K. Lee, B. Su, L. G. Tham, and G. Peter, "Prediction of grout penetration in fractured rocks by numerical simulation," Canadian Geotechnical Journal, vol. 39, no. 6, pp. 1384-1394, 2002.

[32] H. B. Amnieh, M. Masoudi, and M. Karbala, "Evaluating analytical and statistical models in order to estimate effective grouting pressure," Computers and Concrete, vol. 20, no. 3, pp. 275-282, 2017.

[33] Z. Xu, C. Liu, X. Zhou, G. Gao, and X. Feng, "Full-scale physical modelling of fissure grouting in deep underground rocks," Tunnelling and Underground Space Technology, vol. 89, pp. 249-261, 2019.

[34] Z. Zhou, Y. Dong, P. Jiang, D. Han, and T. Liu, "Calculation of pile side friction by multiparameter statistical analysis," Advances in Civil Engineering, vol. 2019, Article ID 2638520, 12 pages, 2019.

[35] X. L. Liu, F. Wang, J. Huang, S. J. Wang, Z. Z. Zhang, and K. Nawnit, "Grout diffusion in silty fine sand stratum with high groundwater level for tunnel construction," Tunnelling and Underground Space Technology, vol. 93, Article ID 103051, 2019.

[36] P. L. Li, Y. Q. Lu, J. X. Lai, and H. Q. Liu, “A comparative study of protective schemes for shield tunneling adjacent to pile groups," Advances in Civil Engineering, vol. 12, Article ID 1874137, 2020.

[37] T. Kasper and G. Meschke, "On the influence of face pressure, grouting pressure and TBM design in soft ground tunnelling," Tunnelling and Underground Space Technology, vol. 21, no. 2, pp. 160-171, 2006.

[38] Y. Liang, J. Zhang, Z. S. Lai, Q. Y. Huang, and L. C. Huang, "Temporal and spatial distribution of the grout pressure and its effects on lining segments during synchronous grouting in shield tunnelling," European Journal of Environmental and Civil Engineering, vol. 21, pp. 1-18, 2017. 
[39] F. Celik, "The observation of permeation grouting method as soil improvement technique with different grout flows models," Geomechanics and Engineering, vol. 17, no. 4, pp. 367-374, 2019.

[40] S. Zhou, X. Zhang, D. Wu, and H. Di, "Mathematical modeling of slurry infiltration and particle dispersion in saturated sand," Transport in Porous Media, vol. 124, no. 1, pp. 91-116, 2018.

[41] F. J. Hou, K. G. Sun, Q. D. Wu, W. P. Xu, and S. J. Ren, "Grout diffusion model in porous media considering the variation in viscosity with time," Advances in Mechanical Engineering, vol. 11, no. 1, Article ID 168781401881989, 2019.

[42] X. Y. Kong, Advanced Mechanics of Fluid Flow in Porous Media, University of Science and Technology of China Press, Beijing, China, 1999.

[43] X. Z. Yang, Study on grout diffusion theory and experiments under static or dynamic loading, Ph.D. Thesis, Central South University, Changsha, China, 2005.

[44] W. J. Ruan, "Research on diffusion of grouting and basic properties of grouts," Chinese Journal of Geotechnical Engineering, vol. 27, no. 1, pp. 69-73, 2005.

[45] Z. Saada, J. Canou, L. Dormieux, J. C. Dupla, and S. Maghous, "Modelling of cement suspension flow in granular porous media," International Journal for Numerical and Analytical Methods in Geomechanics, vol. 29, no. 7, pp. 691-711, 2005.

[46] X. G. Yu, G. H. Xing, and Z. Q. Chang, "Flexural behavior of reinforced concrete beams strengthened with near-surface mounted 7075 aluminum alloys bars," Journal of Building Engineering, vol. 29, Article ID 101086, 2020.

[47] Y. Zhang, D. L. Zhang, Q. Fang et al., "Analytical solutions of non-Darcy seepage of grouted subsea tunnels," Tunnelling and Underground Space Technology, vol. 96, Article ID 103182, 2020. 


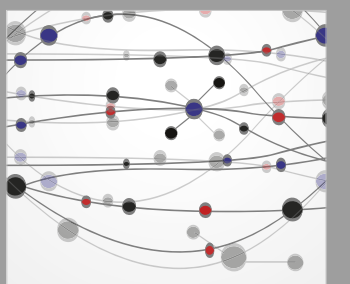

The Scientific World Journal
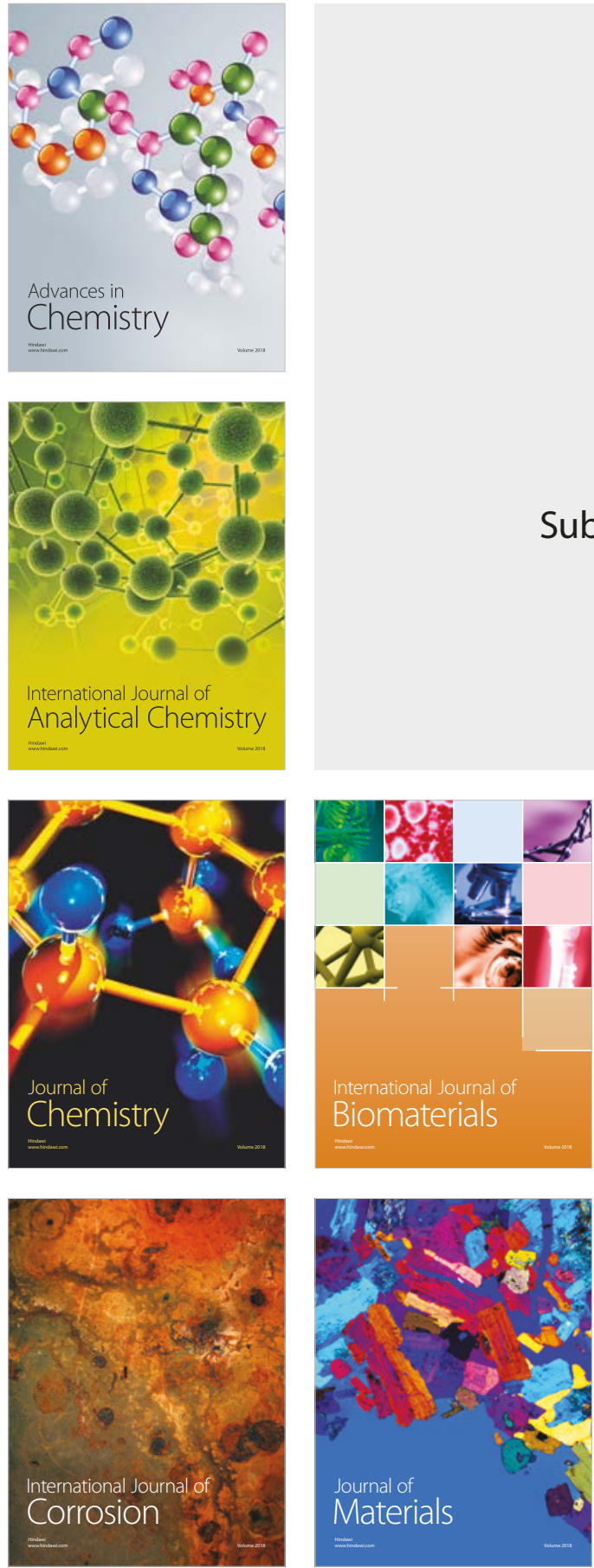

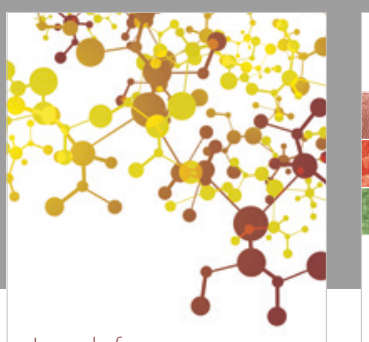

Journal of

Applied Chemistry
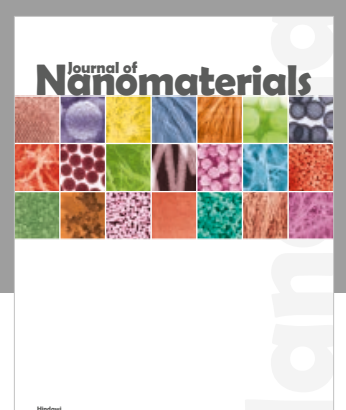

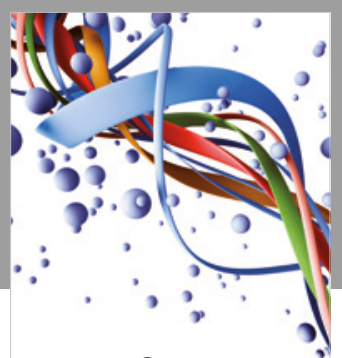

Scientifica

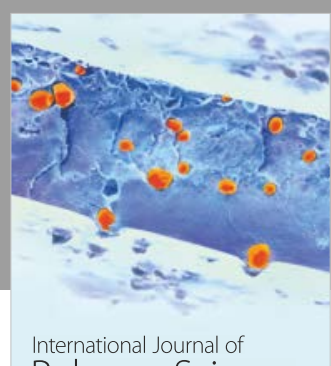

Polymer Science

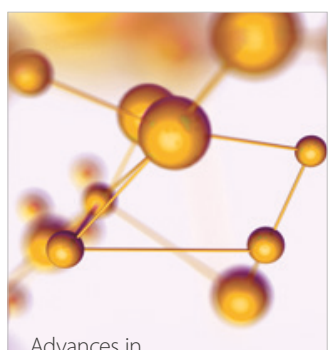

Physical Chemistry
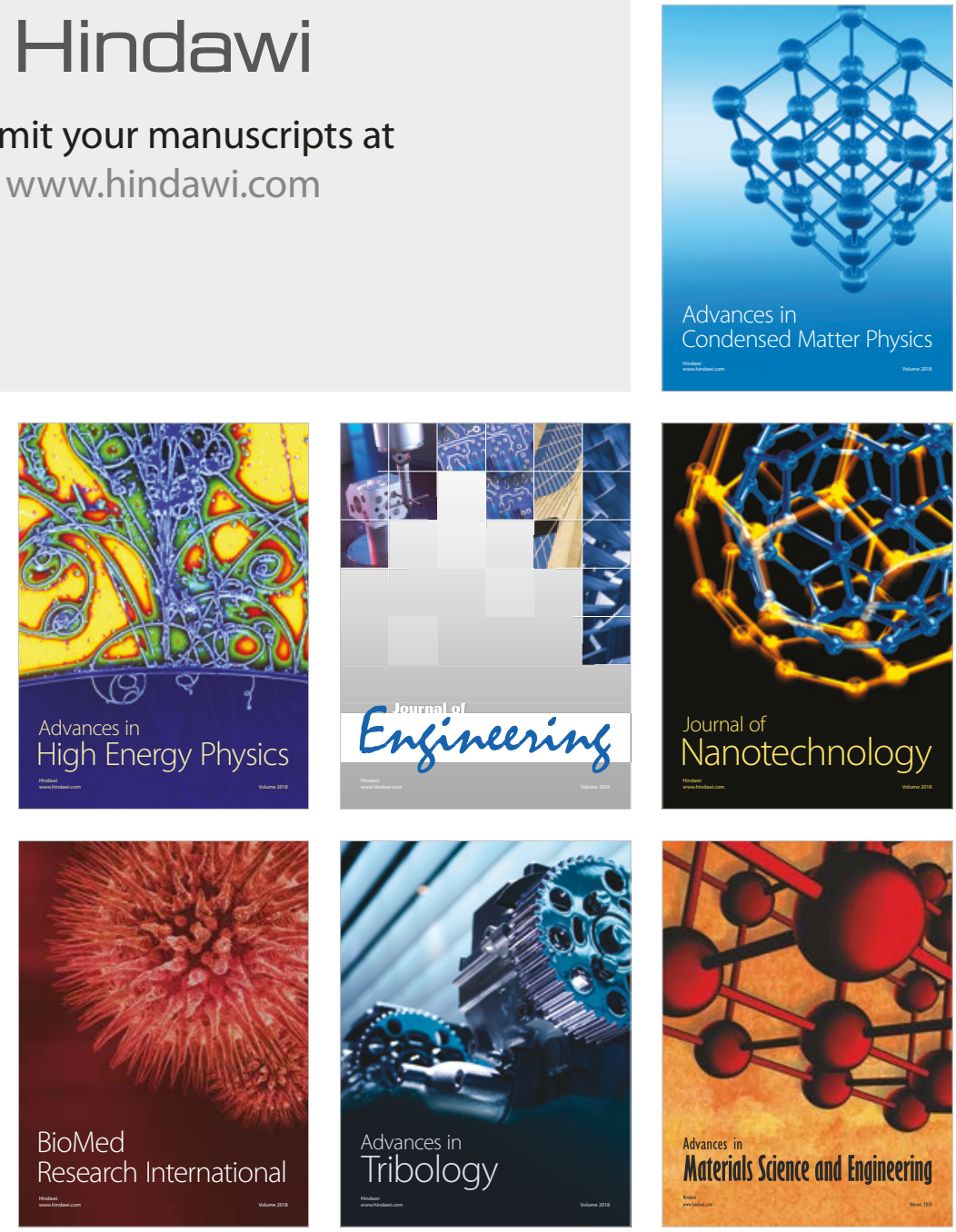